\title{
Recurrent Nodular Fasciitis of the Vulva
}

\author{
Alexander Shushkevich ${ }^{1}$ and Pavel Perevoschikov ${ }^{1}$ \\ ${ }^{1} \mathrm{NN}$ Alexandrov National Cancer Centre
}

June 10, 2021

\begin{abstract}
All vulvar malignancies comprise only $2 \%$ of vulvar lesions, since $98 \%$ of vulvar neoplasms are benign. Nodular Fasciitis of the vulva present a diagnostic dilemma due in part to the rare nature of these disorders and similarities malignant soft tissue tumors, so publishing descriptions of these entities are essential
\end{abstract}

\section{Introduction}

Nodular fasciitis is a self-limited benign clonal process, clinically occurring in the subcutis, composed of fibroblasts and myofibroblasts in a disorganized tissue culture-like array (WHO classification of Skin Tumors, 2018). The most common locations for the disease are the trunk, upper extremities, head, and neck. [1]. Stewart FW first mentioned this disorder in 1944, but Konwaler et al. provided the first details described in 1955 [2]. It has since been described in multiple locations and can be confused with leiomyosarcoma and nerve sheath tumors, so accurate diagnosis is essentially independent of anatomic location.

\section{Case report}

A 41-year-old female presented with a rapidly growing, painful $1 \mathrm{~cm}$ tumor on the right labium major. She underwent surgical excision in November 2017. The tumor was found to be encapsulated, but the capsule was ruptured during the procedure. A provisional diagnosis of leiomyoma was assigned.

She presented again in January 2018 with complaints of a painful mass in the same area. Examination revealed a dense, nodular tumor measuring $5 \times 2 \mathrm{~cm}$ on the right labium majus deep to the prior surgical scar. Pelvic and abdominal ultrasound were routine. Due to clinical concern for malignancy based on rapid regrowth, the patient underwent wide local excision of the right vulva.

During excision, the mass was found to be a solid, well-encapsulated tumor measuring $5 \times 2 \mathrm{~cm}$ with no evidence of any infiltrative process. Grossly negative margins were achieved. The incision was primarily closed.

\section{Investigations}

The initial pathologic consideration of a recurrent tumor was a malignant nerve sheath tumor of the vulva. Due to this tumor's rarity in the vulva, the case was presented at the International Gynecologic Cancer Society monthly videoconference with collaboration from multiple international sub-specialists and pathologists. It was established that the primary and recurrent tumors exhibited similar morphology and presented as a high-cellular spindle cell neoplasm (Figure $1 \mathrm{a}, \mathrm{b}, \mathrm{c}, \mathrm{d}$ ). Immunohistochemistry showed tumor cells stained positive for SMA, calponin, and CD10. Negative for desmin, h-caldesmon, CD117, CD34, HMB-45, BCL-2, cytokeratin, EMA, and p63. CD68 highlighted multinucleated macrophages.

\section{Differential diagnosis}


The diagnosis was reconsidered to be nodular fasciitis based on the cellular morphology and the growth pattern. The tumor consisted of plump fibroblasts with bland nuclear features and a low mitotic rate (4 per $10 \mathrm{HPFs}$, f.d. $0.57 \mathrm{~mm}$ ) in a myxoid and collagenous stroma. Intersecting fascicles with a storiform pattern, high cellular density, pseudocyst formation, and an inflammatory infiltrate containing giant-cell macrophages suggest nodular fasciitis and help narrow the differential diagnosis.

While nodular fasciitis can be suspected based on histologic features, it must be differentiated from fibrosarcoma, smooth muscle tumors, malignant peripheral nerve sheath tumor, dermatofibrosarcoma protuberans, melanoma, and solitary fibrous tumor. Nodular fasciitis has no pathognomonic immunohistochemical markers. However, SMA and vimentin typically show diffuse intensive staining [3]. Desmin is only rarely positive; S100 and h-caldesmon are negative [4-5]. Other positive markers include calponin and CD10 [1,7] (Table 1). Proliferative index Ki67 is highly variable in nodular fasciitis and does not aid in diagnosis.

Fibrosarcoma and malignant smooth muscle tumors are in the differential, but the absence of necrosis, frank nuclear atypia, and atypical mitotic figures support this tumor's benign nature. Immunohistochemistry can aid in differentiating these diagnoses by the presence or absence of desmin, h-caldesmon, and estrogen/progesterone receptor positivity (Table 1). Leiomyosarcoma is positive for desmin and h-caldesmon, but nodular fasciitis is negative for these markers. Fibrosarcoma can be CD34 positive in some cases, usually in dermatofibrosarcoma protuberans, but nodular fasciitis, the positivity is excitingly rare [6].

Malignant peripheral nerve sheath tumor that was initial diagnosis at the time of recurrence was excluded based on the relatively low mitotic count, absence of atypia, and invasion in surrounding tissue with negative S-100 staining on immunohistochemistry. Malignant melanoma was excluded due to the negative S-100 and melan-A staining in this case. Solitary fibrous tumors must show perivascular growth patterns and tend to be positive for CD34 and BCL-2 [8].

\section{Discussion}

Nodular fasciitis is known to be a rear tumor of the vulva. There is only one published case of a recurrence of vulvar nodular fasciitis [8]. This case illustrates the importance of making the distinction between nodular fasciitis and malignant soft tissue tumors, as their differing risks must be matched with appropriate clinical intervention, adjuvant treatment, and follow-up regimen to avoid undertreatment or overtreatment. The pathologist must recognize the possibility of nodular fasciitis even in unusual sites and avoid misdiagnosis of malignancy. This case highlights that recurrence does not always indicate malignancy and can result due to the incomplete initial surgery. The importance of immunohistochemistry is also demonstrated in this case, as histology alone does not exclude other neoplasms in the differential diagnosis (Table 1).

The pathogenesis of nodular fasciitis is unclear, though some suggest antecedent trauma may occur. While the precise incidence in the general population is unknown, it has been described mainly in young and middle-aged adults in the 4 th and 5th decades. Clinically, as in our patient, it is characterized by rapid growth, pain, and size $<3 \mathrm{~cm}[3,9]$.

An important component to optimal health care delivery is the ability to consult experts in related subspecialties or on rare tumors in real time. The monthly international tumor board videoconference facilitated by one gynaecologic oncology professional society, the International Gynaecologic Cancer Society, allows collaboration and accurate diagnosis among gynaecologic oncologists, pathologists, and others. This is a model that may be emulated across the world in various regions and disciplines to improve diagnosis and patient care.

While more common diseases of the vulva include angiomyofibroblastoma, cellular angiofibroma, aggressive angiomyxoma, superficial angiomyxoma, lipoma, and smooth muscle neoplasms (i.e., leiomyoma or leiomyosarcoma) [10], the clinician should recognize that nodular fasciitis may occur in the vulva, and the incidence may be more frequent than previously recognized [8].

Acknowledgements:We would like to acknowledge the International Gynecologic Cancer Society and its ECHO project, in collaboration with the MD Anderson Cancer Center; Anna Plotkin MD (Department of 
Laboratory Medicine and Pathobiology, University of Toronto, Toronto, CA); Jubilee Brown MD (Division of Gynecologic Oncology, Levine Cancer Institute at Atrium Health, Charlotte, NC, USA

Conflict of Interest: The authors report no conflict of interests to declare.

Ethical approval: We have reported this case in compliance with the Declaration of Helsinki. Written informed consent was obtained from the patient.

Data availability statement: The data that support the findings of this study are available from the corresponding author upon reasonable request.

\section{References}

1. Montgomery EA, Meis JM. Nodular fasciitis. Its morphologic spectrum and immunohistochemical profile. Am J Surg Pathol. 1991;15(10):942-8.

2. Konwaler BE, Keasbey L, Kaplan L. Subcutaneous pseudosarcomatous fibromatosis (fasciitis). Am J Clin Pathol 1955; 25: 241-252.

3. Perez-Montiel M, Plaza J, Dominguez-Malagon H. Differential Expression of Smooth Muscle Myosin, Smooth Muscle Actin, H-Caldesmon, and Calponin in the Diagnosis of Myofibroblastic and Smooth Muscle Lesions of Skin and Soft Tissue. Am J Dermatopathol. 2006 Apr;28(2):105-11.

4. Pichler Sekulic S, Sekulic M. Nodular fasciitis of the vulva: a challenging histopathologic diagnosis supported by the detection of USP6 gene rearrangement. APMIS. 2016 Jun;124(6):534-537.

5. Kayaselçuk F, Demirhan B, Kayaselçuk U. Vimentin, smooth muscle actin, desmin, S-100 protein, p53, and estrogen receptor expression in elastofibroma and nodular fasciitis. Ann Diagn Pathol. 2002 Apr;6(2):94-9.

6. Eversole LR, Christensen R, Ficarra G. Nodular fasciitis and solitary fibrous tumor of the oral region: tumors of fibroblast heterogeneity. Oral Surg Oral Med Oral Pathol Oral Radiol Endod. 1999 Apr;87(4):471-476.

7. Hasegawa T, Matsuno Y, Shimoda T. Frequent expression of bcl-2 protein in solitary fibrous tumors. Jpn J Clin Oncol. 1998 Feb;28(2):86-91.

8. Biedrzycki OJ, Singh N, Farugi A. Nodular fasciitis of the vulva with an unusually long clinical history; the importance of making this unexpected diagnosis in such an unusual site. Histopathology. 2007 Oct; 51(4):547-50.

9. Shimizu S, Hashimoto H, Enjoji M. Nodular fasciitis: an analysis of 250 patients. Pathology. 1984 Apr;16(2):161-6.

10. Nucci MR, Fletcher CD. Vulvovaginal soft tissue tumors: update and review. Histopathology. 2000;36(2):97-108. 


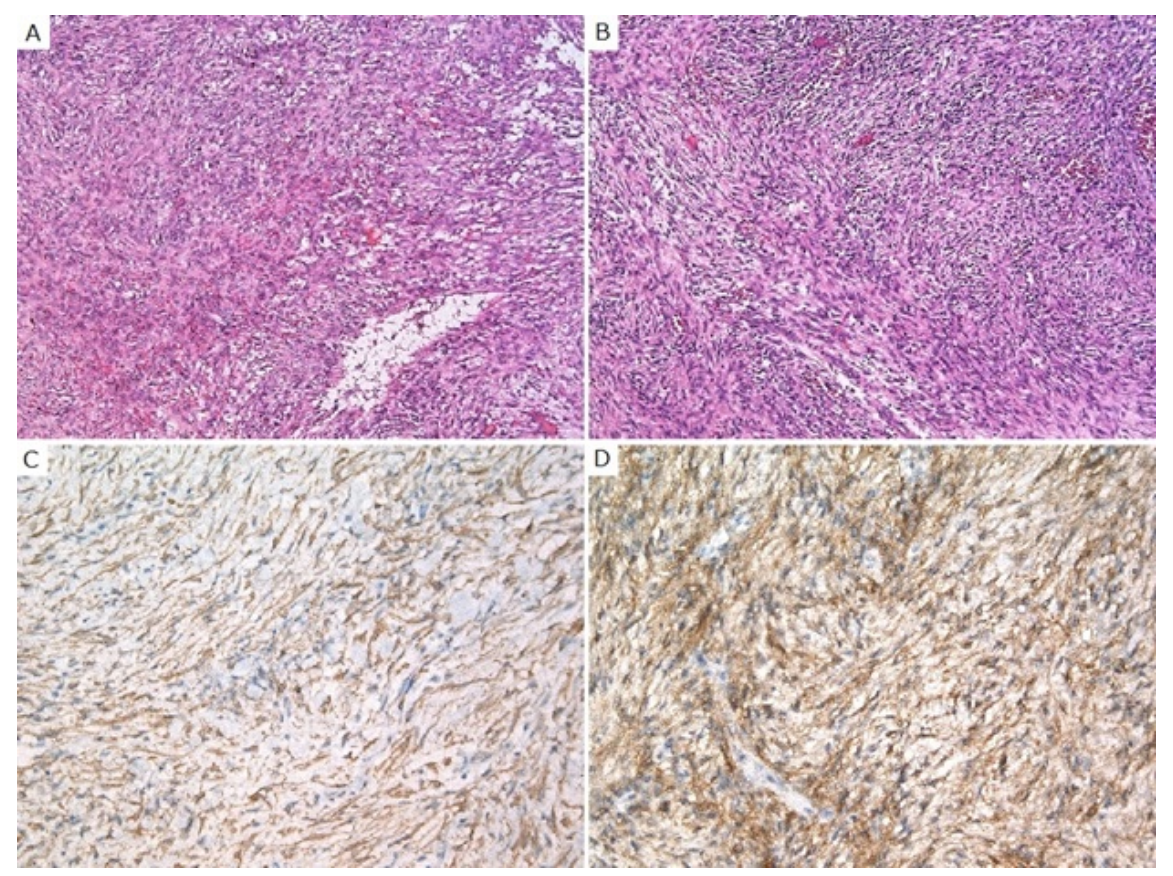

\section{Hosted file}

Table.docx available at https://authorea.com/users/419050/articles/525638-recurrent-nodularfasciitis-of-the-vulva 